\title{
Fiber sensors for the high-speed railway environment
}

\author{
Miguel Gonzalez-Herraez ${ }^{1}$, Massimo Filograno ${ }^{2}$, Pedro Corredera ${ }^{2}$, Alvaro Andres-Alguacil ${ }^{3}$ and Miguel \\ Rodriguez-Plaza ${ }^{3}$ \\ ${ }^{1}$ Departamento de Electrónica, Universidad de Alcalá, Escuela Politécnica Superior, Campus Univ. 28871, Alcala de Henares, Spain. \\ ${ }^{2}$ Instituto de Óptica, CSIC. C/ Serrano 144. 28006. Madrid, Spain. \\ ${ }^{3}$ ADIF, C/Titan 4-6, 28045 Madrid, Spain
}

\begin{abstract}
We review some recent work on fiber-optic sensors for the high-speed railway environment. In particular, we show convenient fiber-optic solutions for rail and wheel monitoring, perimeter security and catenary protection in changeover sections.

OCIS codes: (060.2370) Fiber optic sensors; (060.3735) Fiber Bragg gratings
\end{abstract}

\section{Introduction}

High-speed railway is a fast and energy-efficient mode of transport in continuous growth that needs efficient control and security systems. Conventional monitoring systems in railway infrastructures use electrical sensors that are frequently affected by electromagnetic interferences. In opposition to conventional electrical sensors, fiber-optic sensors assure immunity to electromagnetic fields and simple multiplexing, allowing the use of tens of sensors in a single fiber cable.

In this work, we present the results of field tests concerning the application of fiber optic sensors to the highspeed railway infrastructure, with the final aim of improving the current strategies of maintenance and increasing the safety level of the network. The intrinsic both electrical and magnetic insulation of the optical fiber makes it the best solution for this environment. Moreover, the resistance to a wide range of temperature and to environmental corrosion together with a great sensor multiplexing capability and the low attenuation are additional features that should be considered when applying fiber-optic sensors to the railway monitoring problems.

\section{Rail condition, train and wheel monitoring}

According to recent statistics, the number of passengers will be doubled within 10 years while the volume of goods transported by railway will be tripled. Thus, both axle load and train speed are expected to strongly increase in the next years. In this scenario, wear in the train wheels due to strong braking is also expectable. The most evident consequence of this wear is the appearance of abnormal abrasions in the wheel thread called "flats". Flat spots in the wheel cause strong impacts in the normally smooth power flow from the wheels to the rails. For this reason, wheel flats are a major source of problems in railway systems since they cause strong degradation both in the vehicles and in the infrastructure, they bring noise and discomfort to passengers and they may even cause derailments in extreme cases. These facts put major pressure on the infrastructures and therefore innovative maintaining and inspection techniques are required.

In this work we report on some field tests using Fiber Bragg Gratings (FBGs) for dynamic load measurements in high-speed conditions $(>200 \mathrm{~km} / \mathrm{h})$. These tests are carried out in the Madrid-Barcelona high-speed railway line, with trains running at commercial operation speeds (up to $350 \mathrm{~km} / \mathrm{h}$ ). To install the sensors, a straight sector was selected near the Brihuega High Speed maintenance base, around the KP $69+500$, comprising the transition between an embankment and a trench. Three consecutive spaces between sleepers have been finally instrumented with a total of 11 sensors, as shown in Fig. 1. Of these, sensor P1 is working in pure flexion; P3 and P4 are working in shear, P2 is placed just on the rail neutral line in order to monitor temperature changes in the rail, and sensor P6 measures the vertical compression of the rail in the worst case. The FBG sensors are directly pasted with an epoxy resin on the rail tracks. Prior to installation, the surface of the rail was polished to remove any oxide rests and ensuring that it was not too irregular. The sensors are interrogated using an edge-filter interrogation technique, which allows

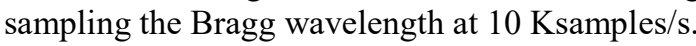




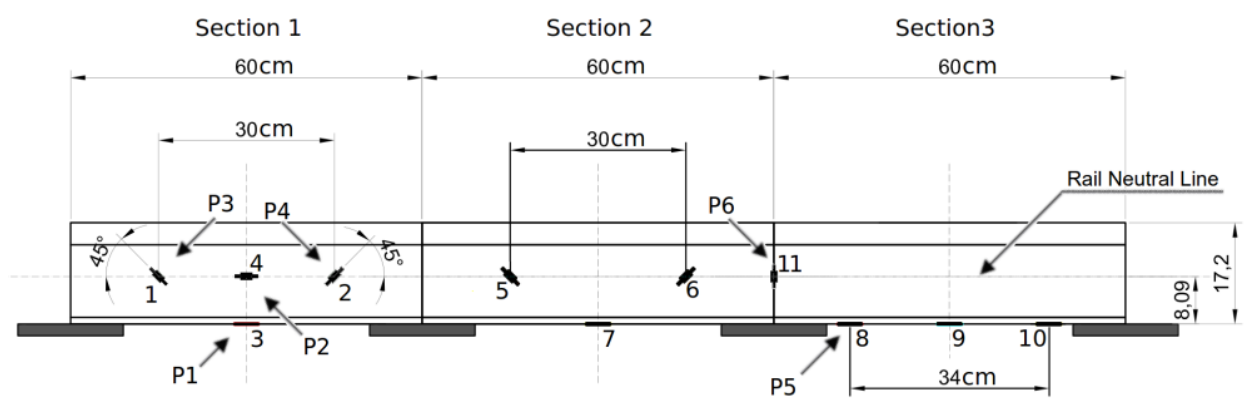

Fig. 1. Disposition of $10 \mathrm{FBG}$ sensors along one of the rails.
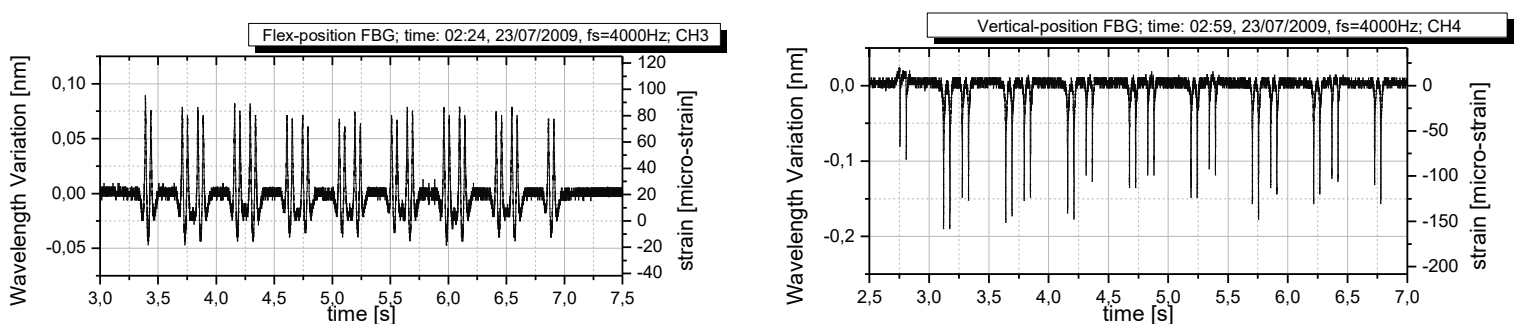

Fig. 2. Strain measured in positions P1 and P6 under the passage of an S103 Siemens-Velaro train.

The results show the potential of this technology for reliable axle load monitoring [1] and wheel flat detection in high-speed railway infrastructures using a reduced set of FBGs installed in the rail [2].

\section{Perimeter security}

Perimeter control is a key issue in high-speed railway lines. Obstacles or intrusions along a high-speed rail may cause severe accidents and often casualties. Tight perimeter control over extremely long distances is therefore necessary. Conventional surveillance systems using cameras are indeed interesting, but these systems are not economically viable to monitor intrusions over distances of $>100 \mathrm{~km}$. Long-range perimeter control is necessary. Our group has made extensive research on phase sensitive reflectometry for intrusion detection, especially over long perimeters [3].

\section{Current monitoring in changeover sections}

A key issue in high-speed railway lines is the protection of the catenaries in changeover sections. Changeover sections are non-fed sections of the catenary that are placed between sections fed with different phases. These sections introduce discontinuities in the current collection done by the train. Whenever the train does not switch-off the current collection before arriving, electric arcs may form and destroy the railway catenary. To avoid these situations, sensors based on Hall effect have been mostly used so far, but they do not provide good isolation at 25 $\mathrm{KV}$ and beyond (or they become extremely expensive). Modern high-speed railways run on these voltage levels, and thus optical current sensors are much more interesting for this application.

In this work, we report on field tests of a polarimetric current sensor based on the Faraday magneto-optic effect for the development of a cost-effective and lightweight all-fibre current sensor. Our setup is specially adapted to the requirements of this application, namely: AC current measurements with high sensitivity, dynamic range up to 500 A, moderate accuracy over the full range, low cost and robustness to environmental and mechanical changes. The setup is electronically self-referenced to account for small misalignments in the polarization and power variations in the optical source [4].

\section{References}

[1] M.L. Filograno, P.Corredera, A. Rodriguez-Barrios, S. Martin-Lopez, M. Rodriguez-Plaza, A. Andres-Alguacil, and M. Gonzalez-Herraez, "Real-Time Monitoring of Railway Traffic Using Fiber Bragg Grating Sensors" IEEE Sensors J. 12, 85-92 (2012)

[2] M.L. Filograno, P.Corredera, M. Rodriguez-Plaza, A. Andres-Alguacil, and M. Gonzalez-Herraez, "Wheel flat detection in high-speed railway systems using Fiber Bragg Gratings" IEEE Sensors J. 13, 4808-4816 (2013)

[3] H. F. Martins, S. Martín-López, P. Corredera, M. L. Filograno, O. Frazão, and M. Gonzalez-Herráez, "Phase-sensitive Optical Time Domain Reflectometer Assisted by First-order Raman Amplification for Distributed Vibration Sensing Over $>100$ km," IEEE J. Lightwave Technol. 32, $1510-1518(2014)$

[4] M. L. Filograno; P. Corredera; M. González-Herráez, " Field testing of a low-cost, self-referenced all-fibre polarimetric current sensor for the monitoring of current in the high-speed railway catenary," in Proceedings of SPIE, 8439, Optical Sensing and Detection II, 84391T (9 May 2012) 